\title{
Residential scene classification for gridded population sampling in developing countries using deep convolutional neural networks on satellite imagery
}

Robert F. Chew ${ }^{1 *}$, Safaa Amer ${ }^{2}$, Kasey Jones ${ }^{1}$, Jennifer Unangst ${ }^{2}$, James Cajka ${ }^{3}$, Justine Allpress ${ }^{3}$ and Mark Bruhn ${ }^{3}$

\begin{abstract}
Background: Conducting surveys in low- and middle-income countries is often challenging because many areas lack a complete sampling frame, have outdated census information, or have limited data available for designing and selecting a representative sample. Geosampling is a probability-based, gridded population sampling method that addresses some of these issues by using geographic information system (GIS) tools to create logistically manageable area units for sampling. GIS grid cells are overlaid to partition a country's existing administrative boundaries into area units that vary in size from $50 \mathrm{~m} \times 50 \mathrm{~m}$ to $150 \mathrm{~m} \times 150 \mathrm{~m}$. To avoid sending interviewers to unoccupied areas, researchers manually classify grid cells as "residential" or "nonresidential"through visual inspection of aerial images. "Nonresidential" units are then excluded from sampling and data collection. This process of manually classifying sampling units has drawbacks since it is labor intensive, prone to human error, and creates the need for simplifying assumptions during calculation of design-based sampling weights. In this paper, we discuss the development of a deep learning classification model to predict whether aerial images are residential or nonresidential, thus reducing manual labor and eliminating the need for simplifying assumptions.
\end{abstract}

Results: On our test sets, the model performs comparable to a human-level baseline in both Nigeria ( $94.5 \%$ accuracy) and Guatemala (96.4\% accuracy), and outperforms baseline machine learning models trained on crowdsourced or remote-sensed geospatial features. Additionally, our findings suggest that this approach can work well in new areas with relatively modest amounts of training data.

Conclusions: Gridded population sampling methods like geosampling are becoming increasingly popular in countries with outdated or inaccurate census data because of their timeliness, flexibility, and cost. Using deep learning models directly on satellite images, we provide a novel method for sample frame construction that identifies residential gridded aerial units. In cases where manual classification of satellite images is used to (1) correct for errors in gridded population data sets or (2) classify grids where population estimates are unavailable, this methodology can help reduce annotation burden with comparable quality to human analysts.

Keywords: Machine learning, Deep learning, Scene classification, Probability based, Complex sample design, Clustering, Remote sensing, GIS

\footnotetext{
*Correspondence: rchew@rti.org

${ }^{1}$ Center for Data Science, RTI International, 3040 East Cornwallis Road,

Research Triangle Park, NC, USA

Full list of author information is available at the end of the article
} 


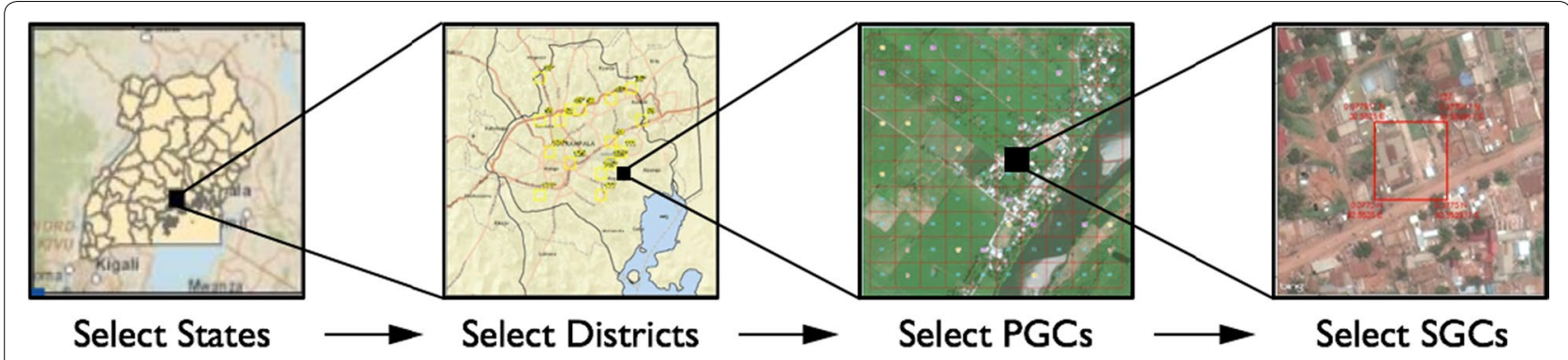

Fig. 1 Overview of an example multistage geosampling design outside of Kampala, Uganda

\section{Background}

Nationally representative survey samples are needed for studies in low- and middle-income countries to support decision-making in research areas ranging from international development to public health. For a probabilitybased sample, this requires an updated sampling frame with adequate coverage of the target population. For a face-to-face survey of households, a country's national census may provide an outdated sampling frame. However, to obtain a statistically efficient probability-based sample of households, an up-to-date roster of households within the sampled area units is necessary. This is often unavailable in low- and middle-income countries, so researchers have traditionally relied on field enumeration of the smallest administrative units or random walk to sample households [1]. In a field enumeration approach, researchers conduct a listing of all households within the sampled areas to construct a sampling frame; the sample of households is then randomly selected from this list. A full listing is time consuming and expensive and requires skilled personnel [2], and it is susceptible to main-street bias (oversampling of highly populous areas), among other errors. In a random-walk approach (also called random route sampling), field staff do not enumerate all households within a selected area; instead, they are provided a starting point and a set of instructions for selecting households while in the field (e.g., sample every fourth house along a specified route). This approach is less resource intensive but lacks statistical rigor because of underlying assumptions about the selection method $[3,4]$, and may be prone to bias because of the effects of interviewer behavior [5-13].

\section{Geosampling for gridded population sampling}

Researchers are developing new and innovative methods that facilitate probability-based survey samples in developing countries at a reasonable cost. One such method, geosampling, uses a geographic information system (GIS) to partition areas of interest into logistically manageable grid cells for sampling [14], contributing to the growing literature on gridded population sampling [2, 15-22]. The first step of geosampling is typically to use a country's administrative geography (e.g., states, districts) from the most recent census to design a multistage probability-based sample up to the smallest administrative unit with reliable information (Fig. 1). Once the smallest available administrative units are sampled, a grid is overlaid on the sampled units to partition them into $1 \mathrm{~km}^{2}$ grid cells, called primary grid cells (PGCs). A probabilitybased sample of PGCs is then selected with the option of integrating population estimate data, derived from GIS resources such as LandScan [23] into the sample design.

The PGCs are further divided into smaller area units called secondary grid cells (SGCs) using a similar approach, albeit without a population estimate at that lower level, and a probability-based sample of SGCs is selected. Using SGCs as the smallest area unit ensures a manageable area size for the field staff conducting data collection, and reduces the degree to which survey respondents are clustered in a particular geographic area. High clustering of sampled units can lead to inflated variance estimates, thus reducing accuracy of survey estimates [24]. Interviewers are then instructed to survey all households within selected SGCs, reducing the potential for interviewer selection bias. Note that SGCs can vary in size ( $50 \mathrm{~m} \times 50 \mathrm{~m}$ to $150 \mathrm{~m} \times 150 \mathrm{~m}$ grid cells) based on population density and urbanicity, rendering smaller areas in dense urban environments and larger areas in more rural settings. This flexibility in grid size variation is designed to help field staff better manage logistics, as large grid areas in population-dense environments make it more difficult for interviewers to effectively scan the entire targeted area, identify households to include in the sample, and attempt to interview all targeted respondents within the grid unit during one visit.

Given the logistical challenges, it is undesirable and costly to send field staff to uninhabited or sparsely populated areas. Prior to sampling PGCs and SGCs, several steps are taken to refine the set of grid cells eligible for selection. First, PGCs with a LandScan population 
estimate lower than 250 people per $\mathrm{km}^{2}$ are excluded from sampling. While gridded population datasets are becoming more detailed [25], population predictions at smaller area sizes have historically been less accurate than at larger geographic units. In particular, case studies have reported large absolute differences existing across gridded population data sets in more populous regions when compared to low density areas [26], and root mean squared error (RMSE) between gridded population estimates and high spatial resolution population census data increasing as the geographic units are more granular [27]. To help mitigate these inaccuracies, a random sample of PGCs with an estimated population greater than 250 people per $\mathrm{km}^{2}$ is selected for visual residential screening. Screening utilizes a human coder who determines if a PGC is residential by using aerial photography to establish the context in which buildings are located. This enables the coder to perceive the likely purpose for the structures. The presentation of residential buildings on an aerial photograph is not uniform within or between communities and countries. It is necessary to consider various geospatial characteristics such as community size, building pattern, and proximity to other land uses when determining whether a building is residential. The final sample of PGCs is selected among those classified as residential. ${ }^{1}$

For SGCs, it becomes difficult to reproduce the screening strategy used for PGCs because LandScan population estimates are not available for SGCs and because the set of SGCs is much larger, increasing the time and cost of screening. Prior applications of geosampling have relied on sequential sampling from a hypergeometric distribution to implement a manageable form of residential screenings for SGCs. A hypergeometric distribution provides the number of successes in sample draws, without replacement, from a finite population of size that contains an exact number of successes (i.e., achieving the draw with the targeted characteristic-in our case, a residential SGC), wherein each draw is either a success or a failure. SGCs are sequentially selected at random, screened for residences, and only enter the sample if deemed residential; this process continues until the desired SGC sample size has been achieved. Because screening ceases before all SGCs within a PGC have been screened, this approach does not provide all the necessary information to calculate appropriate probabilities of selection for residential SGCs. Consequently, a simplifying assumption that the population is uniformly distributed across all SGCs within a PGC must be made during weighting.

\footnotetext{
${ }^{1}$ A residential PGC or SGC is a grid cell with at least one building structure detected in the aerial imagery.
}

\section{Motivation}

Our goal is to create a protocol for how to efficiently and accurately classify SGCs as residential versus nonresidential so that nonresidential grids can be excluded from sampling and accounted for in probabilities of selection. This study assesses the utility of machine learning for this task, as an alternative to manual screening. The advantages of this approach are a reduced level of effort and the ability to create a complete residential screening of all SGCs within sampled PGCs. Furthermore, the availability of complete screening information for SGCs would eliminate the need for simplifying assumptions during calculation of SGC sampling weights. Although geosampling and other methods use satellite imagery for finalstage selection $[2,15-18,20,22,28,29]$, this is the first instance, to the authors' knowledge, of using machine learning to aid in sample frame construction in GIS-enabled sampling methodologies.

\section{Methods for classifying satellite imagery}

The remote sensing community has a long history of detecting geospatial features of interest in satellite imagery. Traditional approaches for feature extraction use spectral properties from individual pixels to determine land use or coverage categories [30-32]. With the wider availability of high-resolution satellite imagery, researchers have expanded to Geographic Object-based Image Analysis (GEOBIA) methods [33-35]. These methods are aimed at identifying and demarcating specific objects of interest, such as lakes or buildings, instead of assigning broad land-cover categories to pixels, such as "water" or "urban."

Increasingly, deep learning models [36] are being used to analyze satellite imagery on diverse tasks, such as semantic segmentation [37], per-pixel classification [38], and poverty mapping [39]. Deep learning has also been particularly successful in scene classification tasks [40-44], which assign an entire aerial image into one of several distinct land-use or land-cover categories. Conceptually, scene classification is equivalent to a binary or multiclass object recognition task in the computer vision literature, except that input images are aerial landscapes instead of portrait or in-profile photographs. As such, our problem can be framed as a two-category scene classification task (predicting whether a satellite image scene is residential vs. nonresidential), where the model results are used to determine which areas are eligible for the survey selection process.

In the following sections, we discuss components of the study, including the data used to test the approach, the machine learning models used for scene classification, and the results. We assess the performance of our deep convolutional neural network (CNN) models 
Table 1 Count of images by grid area sizes in the Nigeria and Guatemala data sets

\begin{tabular}{llcl}
\hline Type & Size & Nigeria & Guatemala \\
\hline PGC & $1 \mathrm{~km} \times 1 \mathrm{~km}$ & 71 & 6 \\
$\mathrm{SGC}$ & $50 \mathrm{~m} \times 50 \mathrm{~m}$ & 410 & 1200 \\
$\mathrm{SGC}$ & $100 \mathrm{~m} \times 100 \mathrm{~m}$ & 3900 & 300 \\
SGC & $150 \mathrm{~m} \times 150 \mathrm{~m}$ & 1044 & 0 \\
\hline
\end{tabular}

against two benchmarks: (1) a human baseline representing the raw agreement between two independent coders, and (2) a machine learning baseline trained on a set of crowdsourced geospatial features from OpenStreetMaps (OSM) and remotely sensed features from the European Space Agency (ESA) Land Cover data set. To better understand the generalizability and reproducibility of our approach, we have tested the models in two different countries-Nigeria and Guatemala-and evaluated the extent to which model accuracy is affected by changes in the training set sample size. Lastly, we conclude with discussions on the approach and future work.

\section{Methods}

\section{Data preparation}

The data used for this study are from two geosamplingbased projects. The first data set is from a random subsample of SGCs from the states of Lagos and Kaduna in Nigeria. All SGCs in the subsample were manually screened and then split to create training and test data sets. An additional data set, which included SGCs from Guatemala City, was used to validate the model's generalizability across different countries and geographic settings. The process of generating the SGC images was the same for both Nigeria and Guatemala. Table 1 summarizes the different grid areas sizes for the Nigeria and Guatemala data sets, respectively.

Aerial and satellite images were retrieved through three web map services, providing global access to recent Google, Bing, and Esri base maps and imagery. Gridbased polygon layers for both PGCs and SGCs were constructed in ArcGIS, and the source of the imagery at the time of the survey was recorded for future reproducibility. Google and Bing image services are commercially available to ArcGIS users for a modest license fee, and Esri imagery is natively integrated into the GIS software. While these tiled image services provide worldwide coverage, they can vary in both age and spatial resolution from 1 to $2 \mathrm{~m}$ depending on the specific geographic location. As such, the imagery provided by each of these services may differ in resolution, color balance, brightness, and cloud cover from location to location, and between vendors. To help determine the best imagery for identifying residential areas for a given location, a graphical user interface (GUI) was developed to help human coders toggle between the different imagery services while classifying grids as residential versus nonresidential (see "Gold-standard labels" section for more detail on developing gold-standard labels). Although using different imagery sources complicates the analysis, it exposes the methodology to implementation scenarios that research teams may realistically encounter. Model performance across imagery sources and grid area sizes are presented in the Results.

We selected 71 random PGCs in Nigeria that contained residential development (Fig. 2a), as well as an additional 6 PGCs in Guatemala (Fig. 2b). Because of the relatively smaller sample size in Guatemala, diversity in urbanicity and geographical characteristics were considered for PGC selection instead of a purely random selection to ensure better generalizability.

From these PGCs, 5350 SGC images were created for Nigeria and 1500 for Guatemala. The size of the secondary grid unit was determined by its level of urbanicity as defined from the latest country census. Urban areas had smaller grid cells than rural areas to account for population density, to avoid high clustering, and so that field staff would have a more consistent workload across SGCs.

Although this adds complexity to the modeling task, we included it in the study to more realistically mirror survey field work considerations. The Nigerian images were composed of 410 grid cells of $50 \mathrm{~m} \times 50 \mathrm{~m}, 3896$ grid cells of $100 \mathrm{~m} \times 100 \mathrm{~m}$, and 1044 grid cells of $150 \mathrm{~m} \times 150 \mathrm{~m}$ images. The Guatemalan set was composed of 1200 grid cells of $50 \mathrm{~m} \times 50 \mathrm{~m}$ and 300 grid cells of $100 \mathrm{~m} \times 100 \mathrm{~m}$ images. Figure 3a shows an example SGC grid in Nigeria and Fig. 3b shows an example SGC grid in Guatemala.

\section{Labelling data \\ Gold-standard labels}

To develop the gold-standard labels of whether a grid is considered "residential" or "nonresidential," SGCs were individually evaluated by coders to determine if they contained one or more buildings within the image. If the image contained one or more buildings, the entire grid was considered "residential"; otherwise, it was considered "nonresidential." Since there is a certain amount of subjective decision making required by the coders to determine if buildings are present, the data were labelled by two independent coders, with a senior GIS analyst acting as an adjudicator to settle disputed labels and to ensure consistency and accuracy in selection. The instances of coder disagreement were the motivation for the human benchmark metric ("Human benchmark metric" section) and is further examined in the "Discussion" section. 


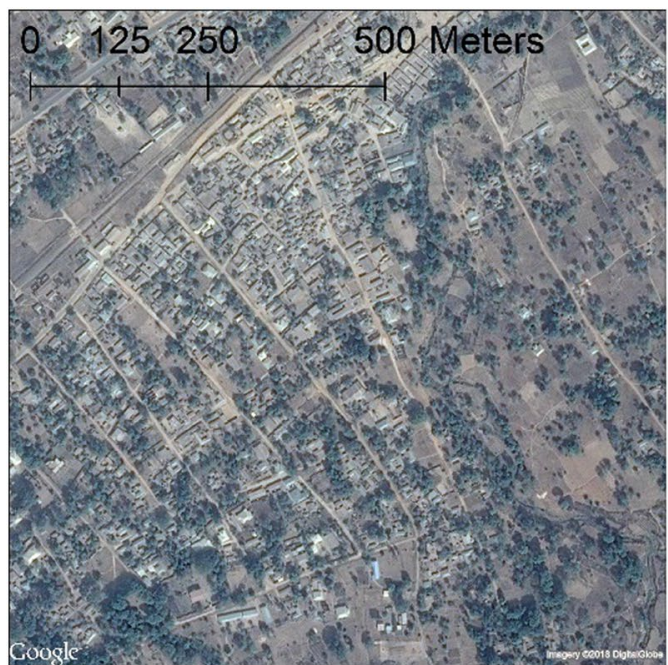

a

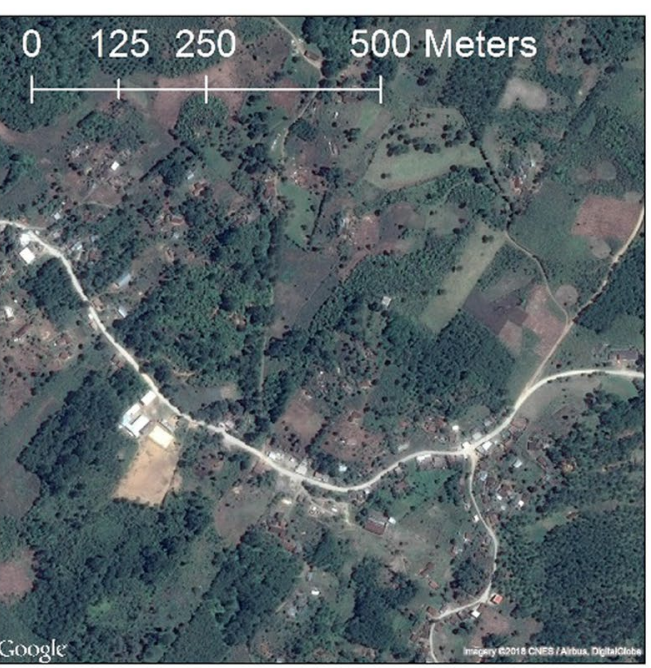

b

Fig. 2 a Nigeria PGC Image (1 km × $1 \mathrm{~km})$. b Guatemala PGC Image $(1 \mathrm{~km} \times 1 \mathrm{~km})$

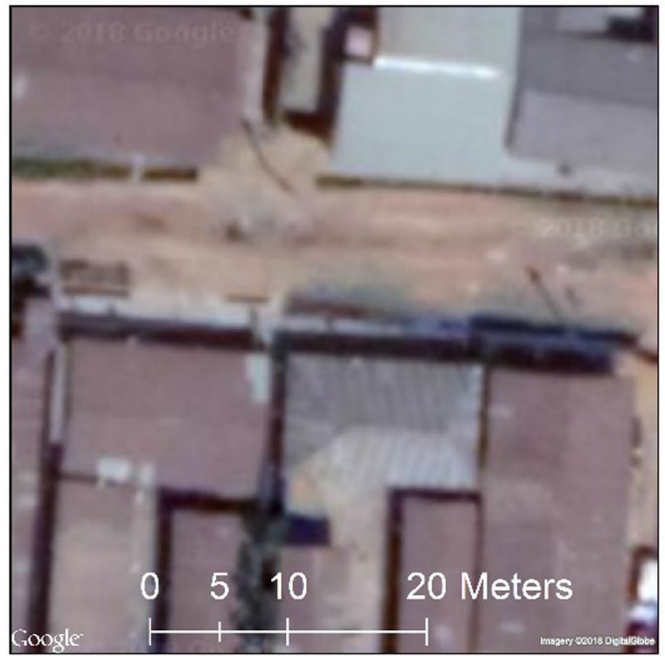

a

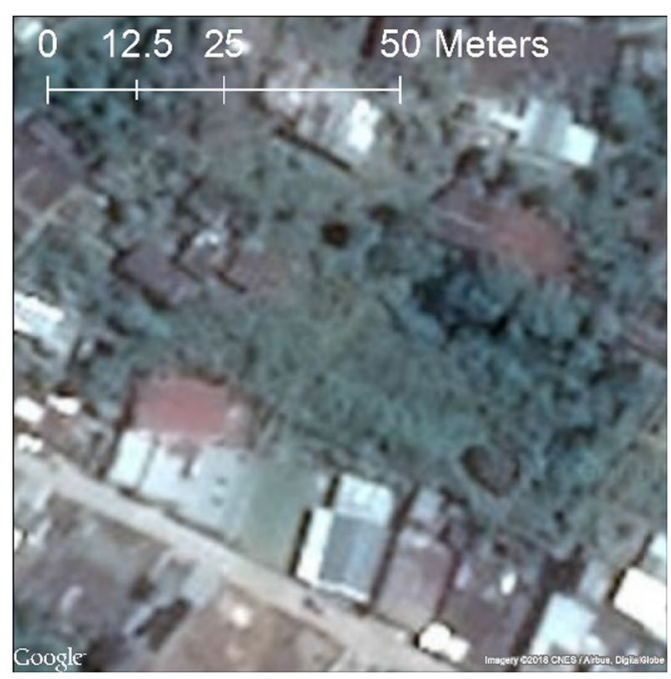

b

Fig. 3 a Nigeria SGC Image $(50 \mathrm{~m} \times 50 \mathrm{~m})$. b Guatemala SGC Image $(100 \mathrm{~m} \times 100 \mathrm{~m})$

This process was completed using a GUI tool developed within ArcGIS for applying a residential or nonresidential label to each of the grid cells. Figure 4a provides an example residential SGC image whereas Fig. 4b presents an example nonresidential image.

\section{Human benchmark metric}

To provide a naïve human-level benchmark for how consistently coders agree on labels for this task, we computed the raw agreement [45] between our two independent coders, prior to adjudication. The raw agreement for two coders can be calculated using the following formula:

$$
\text { Raw Agreement }=\frac{1}{N} \sum_{i=1}^{C} n_{i i}
$$

where $N$ is the total number of images that are jointly labelled by the two coders, $n_{i j}$ is the number of cases assigned as $i$ by Coder 1 and $j$ by Coder 2 for categories $i$, 


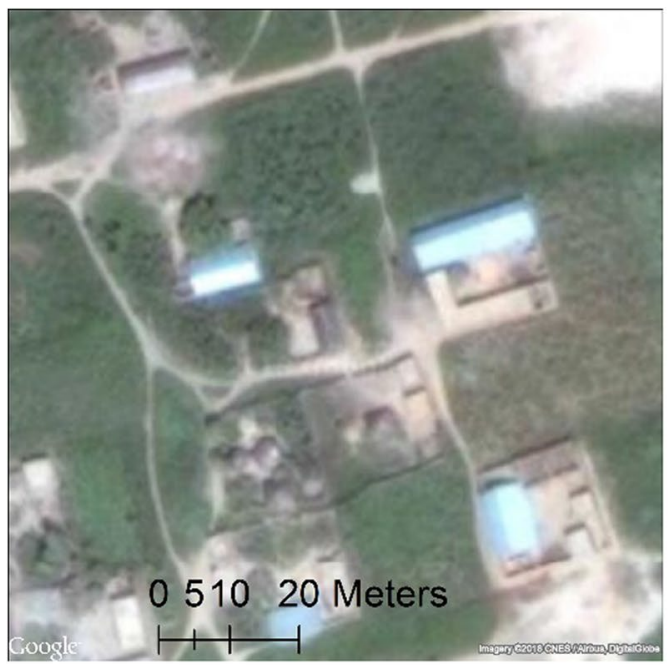

a

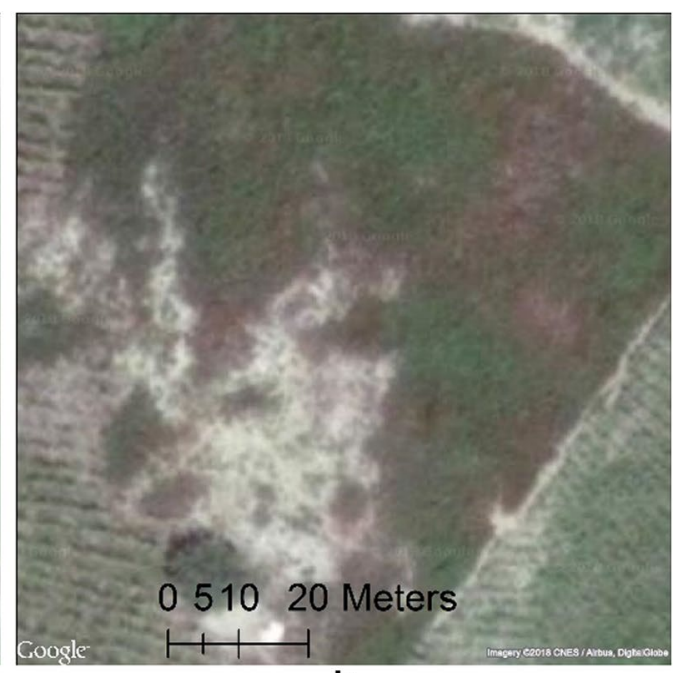

b

Fig. 4 a Example SGC residential scene $(100 \mathrm{~m} \times 100 \mathrm{~m})$. b Example SGC nonresidential scene $(100 \mathrm{~m} \times 100 \mathrm{~m})$

$j=1, \ldots, C$ and $C$ is the total number of categories (in our case, residential and nonresidential).

Although other measures of inter-rater reliability have been developed to correct for when coder agreement occurs by chance $[46,47]$, there are several benefits to using raw agreement for comparison. First, it provides the cleanest comparison to classification model predictions, because it is mathematically equivalent to the "overall accuracy" evaluation metric commonly used in scene classification tasks. The only distinction between the two is that raw agreement compares the difference in labels between two humans, whereas classification accuracy typically compares the difference between a goldstandard human label and a model prediction. Second, inter-rater reliability measures that account for agreement that is expected to occur through chance, such as Cohen's kappa, can be controversial depending on the context. In the social and health sciences, Cohen's kappa has been criticized for (1) its "base rate problem" [48], the difficulty in comparing kappa statistics across studies due to the statistic's dependence on the proportions of positive and negative examples in any given sample, and (2) the assumptions the statistic inherently makes about the decision-making process of raters, which should instead be explicitly modeled for each rater individually [49]. In the remote sensing community, the kappa statistic has been heavily criticized for its use in assessing land change, being scrutinized for reasons such as its assumption of randomness being an irrelevant baseline for many spatial classification problems [50] and being redundant, since it is highly correlated with overall accuracy [51].
For these reasons, raw agreement was used in this study over other reliability metrics, although additional evaluation measures were used to assess model performance ("Model evaluation" section).

Of the 5350 Nigerian images, coders disagreed on labels for 482 grids, resulting in a raw agreement of $91.0 \%$. Of the 1500 Guatemalan images, coders disagreed on 44 grids, resulting in a raw agreement of $97.1 \%$.

\section{Training and test sets}

The Nigeria and Guatemala data were randomly split into training sets for building models (85\%) and test sets for model evaluation (15\%), stratified to preserve the class ratios of residential and nonresidential images found in the overall data. Although not severely unbalanced, nonresidential grids were more common than residential grids in both our Nigeria (63/37) and Guatemala (67/33) samples. Table 2 provides a breakdown of the training and test sets, respectively, by country and class type.

Table 2 Training and test data set allocation for Nigeria and Guatemala

\begin{tabular}{lcc}
\hline & Nigeria & Guatemala \\
\hline Training set & 4550 & 1275 \\
Residential & 1676 & 417 \\
Nonresidential & 2874 & 858 \\
Test set & 800 & 225 \\
Residential & 295 & 73 \\
Nonresidential & 505 & 152 \\
Total & 5350 & 1500 \\
\hline
\end{tabular}


As of writing, most open source machine learning libraries do not support modeling.tiff files, so the images were converted to.png format. Additionally, the images were rescaled from $720 p \times 720 p$ to $150 p \times 150 p$ for computational efficiencies, as smaller images allow for faster model training and easier handling of large batch sizes. When applicable, we performed additional pre-processing steps for the pre-trained models assessed for transfer learning, as specified in the original papers $[52,53]$. These steps are necessary to ensure that the models produce reliable output by matching the input data format used to originally train the models.

\section{Residential scene classification models}

To create a model that can accurately discern between residential and nonresidential aerial images, we develop a series of scene classification models based on machine learning methods. Machine learning is a subdiscipline of artificial intelligence that focuses on the ability of computers to "learn" how to perform tasks without being explicitly programmed how to do so. For example, rather than hand-code software routines with specific instructions on how to identify residential scenes from images, a model is "trained" to learn how to distinguish between residential and nonresidential scenes from examples of labelled data. Exploring modern machine learning methods for aerial scene classification is attractive due to the near human-level performance they have achieved in tasks as diverse as object recognition [54-56], speech recognition [57, 58], and gaming [59-61]. Additionally, after a model is trained, predicting the classes of new images can be automated without additional human intervention and performed at scale. For the use case of screening grids for residential or nonresidential scenes, these models can be used as the sole screening tool or as an additional quality check to assist a team of human annotators.

The scene classification models presented in this paper can be classified into two overarching groups: (1) "deep learning" models [36], which learn data representations by processing raw data inputs through multiple successive model layers that detect features (most commonly, performed with artificial neural network models) and (2) more traditional "shallow learning" models that learn decision rules from variables (i.e., features) created by modelers with expertise or experience with the phenomena being modeled. In our case, we develop deep learning scene classification models directly from labelled satellite images without explicitly creating variables that distinguish between residential and nonresidential grids. These models are described in the "Deep learning models" section. For comparison, we also develop shallow learning scene classification models with analyst-derived features from the open global GIS datasets OSM [86] and the European Scape Agencies Climate Change Initiative project [62]. These models are described further in the "Shallow learning models" section. A workflow diagram of the two sets of modeling approaches is also included in Fig. 5. In total, there are 11 models developed for Nigeria and another 11 models for Guatemala, whose predictions are compared with each other on the test sets and to the human coder raw agreement scores. Testing such a large number of models is motivated by the No Free Lunch Theorem [63], which states that there are no theoretical guarantees that any one standard machine learning algorithm will work best on all tasks, implicitly promoting an empirical approach to model selection for supervised classification problems.

\section{Deep learning models}

Baseline convolutional neural network As a baseline deep learning model, we constructed an eight-layered convolutional neural network $(\mathrm{CNN})$ consisting of three convolutional, three pooling, and two fully connected layers. A CNN is a type of artificial neural network model that contains a convolution filter as at least one of its layers. In image processing, a convolution filter (or kernel) is a small matrix of values that, when applied to a larger image, can help isolate notable image features (edges, corners, etc.). Convolution filters use the convolution matrix operation to extract features, often convolving the filter across the image in a sliding window to capture local details. While researchers have developed many specialized filters for feature extraction $[64,65], \mathrm{CNN}$ filters are not specified a priori to extract any specific features. Instead, elements of the CNN filter matrix are included as model parameters and derived during the training process, effectively creating custom filters salient for the specific modeling task. Deep CNNs take this a step further by chaining convolution layers together, a process that ideally captures increasingly higher-level and more nuanced representations of the data. This model uses $3 \times 3$ convolution filters with a stride of 1 to extract data representations.

Other types of layers besides convolutional layers are often included in CNNs to perform complementary actions. Max pooling [66] was performed in three layers with a $2 \times 2$ filter to reduce the number of parameters and help prevent overfitting. Max pooling is a simple dimension reduction technique in which a portion of a matrix is isolated and the max value of the isolated elements is returned. This simplified representation summarizes characteristics of the earlier layers, helping later layers generalize more broadly rather than learn traits that are specific only to a particular image. In addition, rectified linear units (ReLU) were used for the activation function to speed up training [67]. Activation functions serve the 


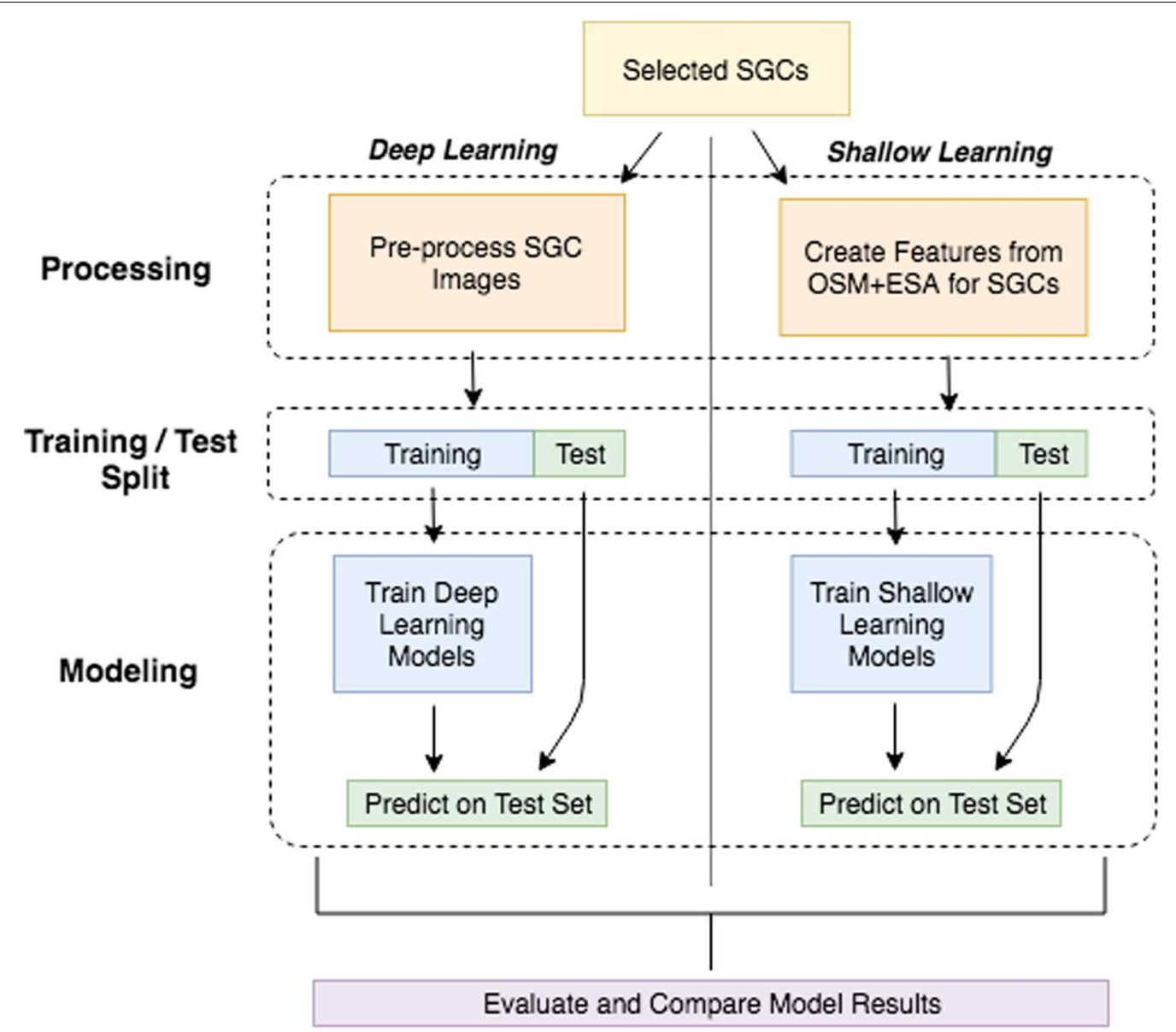

Fig. 5 Workflow diagram of modeling approach

same purpose as link functions for general linear models (GLMs) in the statistics literature [68], providing a way of transforming a linear predictor into a nonlinear response space. ReLUs differ from other popular activation functions like the logistic sigmoid function (commonly used in logistic regression) in that ReLUs return zero at any input values in the negative domain and return the input value itself in the positive domain:

$$
f(x)=x^{+}=\max (0, x)
$$

The first fully connected dense layer also used a ReLU activation function and leveraged a dropout method $[\mathrm{p}($ dropout $)=0.5]$ to prevent overfitting [69]. Dropout is a regularization technique in which units in your neural network are randomly dropped (along with their connections) during training. The intuition behind this method is that, by thinning the network connections in your fully connected layers, you prevent parameters from being too interdependent among themselves, resulting in a network that will generalize better to new examples. A final dense layer with a sigmoid activation function is used to create predicted probabilities of inclusion for either the "residential" or "nonresidential" classes. The model was run with a batch size of 25 images each and trained over 35 epochs. Figure 6 presents a simplified network diagram of the baseline CNN.

Transfer learning Large labelled data sets or strong regularization are often required to effectively train deep learning models without overfitting [69]. While many state-of-the-art deep learning models have dozens of layers $[52,53]$, this can results in thousands or even millions of model parameters to fit. Training an exceedingly deep architecture from scratch with random initializations was prohibitive for our sample size, so we used a transfer learning approach [70-72] to leverage stable weights from deep CNN classification models trained on much larger data sets. Transfer learning is a learning framework in which the objective is to use a model trained in one source domain (or task) to help build a model in a related target domain (or task) without the need for considerable new labelled data [70]. 


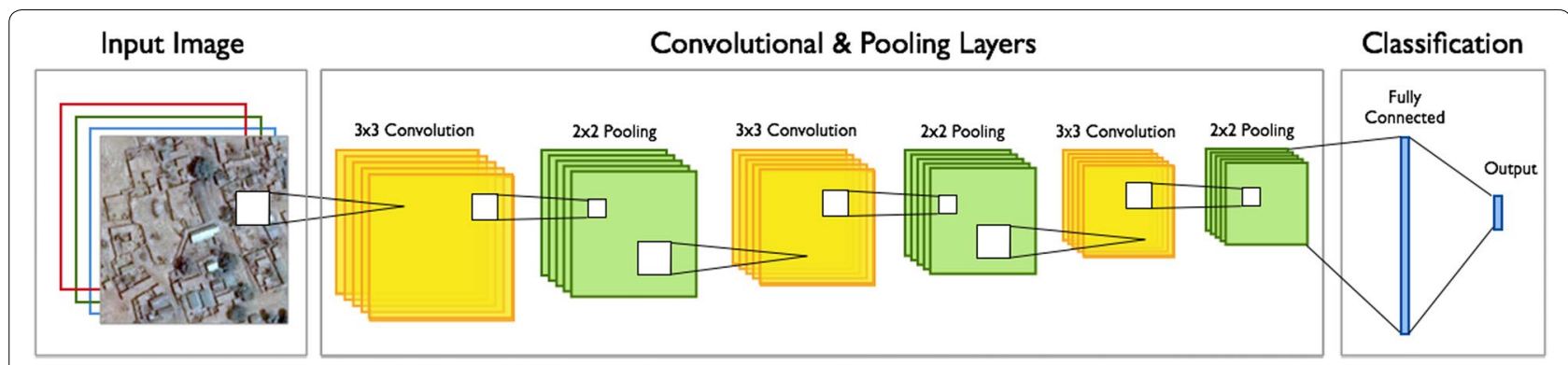

Fig. 6 Network diagram of baseline CNN

This "knowledge transfer" paradigm, in which general features learned from one task help inform a similar task, has become particularly popular with deep CNNs, as pretrained models built on large labelled datasets are often available through open source code repositories.

To test a transfer learning approach, we used the ImageNet dataset as our source domain and the labelled grid scene images as the target domain. ImageNet is a labelled image data set consisting of over 1.2 million high-resolution images and 1000 categories [73], which were collected from the web and labelled by human coders on Amazon's Mechanical Turk platform. ImageNet categories are based off the lexical database WordNet, which semantically organizes and groups commonly used words into concept hierarchies [74]. As such, ImageNet does not include aerial images because they are not generally associated with archetypical representations of objects (e.g., a standard image of a building would be more likely to be portrayed in profile or as part of a landscape rather than from an overhead view). In addition, aerial images may contain many distinct objects in the same image whereas ImageNet images do not. Even with this limitation, transfer learning with ImageNet trained models have produced stateof-the-art results on images that do not fit this criteria, such as medical [75] and satellite imagery [76].

While it seems unintuitive that a model built on nonaerial images could help develop a model that identifies residential gridded aerial units, deep CNNs have been shown to benefit from spatial hierarchies of patterns [77] in which earlier layers detect small localized patterns (such as edges), while later layers construct more complex representations (such as shapes) composed of the localized patterns detected in earlier layers. While complex representations at later layers can reduce the performance of transfer learning to new tasks if they are too highly specialized [72], research suggests that transferring features even from dissimilar tasks can be better than using random parameter initializations [72]. In addition, transferability tends to increase as the similarity between tasks increases; [72] as such, we favor using pretrained model weights in this study that were originally trained to solve a task similar to ours (Inception V3 [52] and VGG16 [53] used for object recognition).

To test the viability of transfer learning, we used pretrained models from the well-known Inception V3 [52] and VGG16 [53] architectures. Inception and VGG16 are deep CNN model architectures that won first and second place, respectively, at the ImageNet Large-Scale Visual Recognition Challenge 2014 (ILSVRC 2014) and have been used successfully for transfer learning on tasks diverse as cell nuclei classification on histopathology images [78], human aquatic activities classification on micro-Doppler signatures [79], and fruit detection in orchards [80]. Model parameters (i.e., weights) for the architectures trained on ImageNet were acquired through the Python Keras library implementation [81]. To allow the pretrained weights to update for our modeling task, we performed transfer learning in two steps. First, we ran our training and test images through the pretrained Inception V3 and VGG16 networks on all but the top layers, which often consist of a fully connected layer to flatten the dimensionality and an evaluative fully connected layer with a softmax activation function to provide predicted probabilities for class assignment. The top layers of the pretrained models were not included, because we are not interested in predicting the original ImageNet classes. Second, we used the resulting "bottleneck features" [82] as the base for training our own small fully connected model with our classes of interest (residential vs. nonresidential). Our model includes a fully connected layer with ReLU activation units, a dropout layer with a probability of dropout $=0.5$, and a final output layer with a sigmoid activation function to produce class probabilities.

As a final experiment, we created an ensemble model [83-85] of our transfer learning models by averaging each model's predicted probabilities. The premise behind ensemble learning is that a diverse set of models can 
Table 3 GIS derived OSM + ESA variables

\begin{tabular}{llll}
\hline Variable name & Type & Number & Description \\
\hline ContainBuildings & Binary & 1 & Whether an SGC contains an OSM building polygon \\
SemiFitBuild & Binary & 1 & Whether an SGC contains a semi-filtered OSM building polygon \\
AnyRoad & Binary & 1 & Whether the SGC intersects any OSM road \\
ResRoad & Binary & 1 & Whether the SGC intersects any OSM road labelled residential \\
ResPlusUnRoad & Binary & 1 & Whether the SGC intersects any OSM road labelled residential or unlabeled \\
Glob2015_MajLC & Categorical & 38 & ESA land-cover categories, ranging from "cropland" to "permanent snow and ice" \\
\hline
\end{tabular}

achieve better predictive performance than any of the individual constituent models alone.

\section{Shallow learning models}

Although aerial and satellite images provide a direct way of detecting remote land features, modeling on aerial images is unnecessary if the features of interest are already captured in existing data sets. Large, open geospatial databases, such as OSM [86], provide crowdsourced annotations of roads and buildings for areas worldwide. Furthermore, open data sets of land cover categories, maintained by ESA used to study the effects of climate change [62], provide land use and development patterns. As an additional benchmark, we developed classification models using data derived from OSM and ESA to compare the effectiveness of object recognition models using aerial satellite imagery to classification models using features derived from open geospatial databases. To ensure that the methods could be reproduced in new countries, we only considered data sources that were both open source/freely available and had a global scope.

Table 3 provides a list of variables created for the OSM+ESA data set. These variables were assigned to each PGC and SGC by intersecting the grid cell boundaries and the various contributing geospatial layers using ArcGIS. Building and road features were extracted from OSM while major land-cover variables were assigned to the grid cells from the ESA Climate Change Initiative project. The intersection of buildings to grid cell boundaries was performed twice. The first analysis determined if a grid cell contained any building while the second intersection only included buildings that were not classified by OSM as having a non-residential use. Examples of non-residential buildings that were excluded from the intersection include churches, stores, and banks. This variable within the dataset is referred to as semi-filtered as OSM building data is not comprehensively attributed. The classification of grid cells using ESA data assigned each SGC with the land cover classification that intersected the largest proportion of the grid cell.
We assessed the OSM+ESM data set on seven different classifiers (decision trees, gradient boosting trees, AdaBoost, random forest, logistic regression, support vector machines, and k-nearest neighbors) using the scikit-learn package in Python [87]. The models were run for both Nigeria and Guatemala using the same training and test splits as the deep CNN models for comparability.

\section{Model evaluation}

To evaluate model performance on the test set, we used the following four metrics to assess different aspects of the predictions:

1. Overall accuracy-percent of correct predictions.

2. Precision-true positives/(true positives + false positives). Indicates the number of true positives out of all observations that are predicted positive (i.e., of all the grids that are predicted residential, the percentage that are actually residential).

3. Recall-true positives/(true positives + false negatives). Indicates the number of true positives detected (i.e., the percentage of all residential grids predicted residential by the model).

4. F1-score-harmonic mean of precision and recall:

$$
F_{1}=\frac{2}{\frac{1}{\text { recall }}+\frac{1}{\text { precision }}}
$$

These metrics were calculated for each model evaluated, on both data sets. For additional model assessments, we compared overall accuracy across imagery sources (Google, Bing, Esri) and SGC grid area sizes (50 m $\times 50 \mathrm{~m}, 100 \mathrm{~m} \times 100 \mathrm{~m}, 150 \mathrm{~m} \times 150 \mathrm{~m}$ ). Last, we tested the model sensitivity with respect to the amount new of training data required, to better understand the expected data annotation burden on future survey projects. 
Table 4 Model evaluation metrics for the Nigeria and Guatemala test sets

\begin{tabular}{|c|c|c|c|c|c|}
\hline Model & Type & Acc. & Prec. & Recall & F1 \\
\hline \multicolumn{6}{|l|}{ Nigeria } \\
\hline Baseline CNN & Deep & $88.9 \%$ & $89.2 \%$ & $88.9 \%$ & $89.0 \%$ \\
\hline VGG16 with ImageNet weights & Deep & $93.4 \%$ & $93.4 \%$ & $93.4 \%$ & $93.3 \%$ \\
\hline InceptionV3 with ImageNet weights & Deep & $93.6 \%$ & $93.6 \%$ & $93.6 \%$ & $93.6 \%$ \\
\hline VGG16 and InceptionV3 ensemble & Deep & $94.5 \%$ & $94.5 \%$ & $94.5 \%$ & $94.5 \%$ \\
\hline Decision Tree & Shallow & $80.3 \%$ & $80.9 \%$ & $80.3 \%$ & $78.9 \%$ \\
\hline Gradient Boosting & Shallow & $80.3 \%$ & $80.9 \%$ & $80.3 \%$ & $79.0 \%$ \\
\hline AdaBoost & Shallow & $80.6 \%$ & $81.8 \%$ & $80.6 \%$ & $79.2 \%$ \\
\hline Random forest & Shallow & $80.1 \%$ & $80.7 \%$ & $80.1 \%$ & $78.8 \%$ \\
\hline Logistic regression & Shallow & $80.6 \%$ & $81.8 \%$ & $80.6 \%$ & $79.2 \%$ \\
\hline Support vector machine & Shallow & $79.9 \%$ & $81.5 \%$ & $79.9 \%$ & $78.1 \%$ \\
\hline K-nearest neighbors & Shallow & $75.6 \%$ & $81.3 \%$ & $75.6 \%$ & $71.3 \%$ \\
\hline Human benchmark & Human & $91.0 \% *$ & - & - & - \\
\hline \multicolumn{6}{|l|}{ Guatemala } \\
\hline Baseline CNN & Deep & $93.3 \%$ & $93.3 \%$ & $93.3 \%$ & $93.3 \%$ \\
\hline VGG16 with ImageNet weights & Deep & $96.4 \%$ & $96.7 \%$ & $96.4 \%$ & $96.5 \%$ \\
\hline Inception V3 with ImageNet weights & Deep & $95.6 \%$ & $95.9 \%$ & $95.6 \%$ & $95.6 \%$ \\
\hline VGG16 and InceptionV3 ensemble & Deep & $96.4 \%$ & $96.7 \%$ & $96.4 \%$ & $96.5 \%$ \\
\hline Decision tree & Shallow & $93.8 \%$ & $94.1 \%$ & $93.8 \%$ & $93.8 \%$ \\
\hline Gradient boosting & Shallow & $93.8 \%$ & $94.1 \%$ & $93.8 \%$ & $93.8 \%$ \\
\hline AdaBoost & Shallow & $92.9 \%$ & $93.1 \%$ & $92.9 \%$ & $93.0 \%$ \\
\hline Random forest & Shallow & $93.8 \%$ & $94.1 \%$ & $93.8 \%$ & $93.8 \%$ \\
\hline Logistic regression & Shallow & $93.8 \%$ & $94.1 \%$ & $93.8 \%$ & $93.8 \%$ \\
\hline Support vector machine & Shallow & $93.8 \%$ & $94.6 \%$ & $93.8 \%$ & $93.9 \%$ \\
\hline K-nearest neighbors & Shallow & $92.4 \%$ & $93.7 \%$ & $92.4 \%$ & $92.6 \%$ \\
\hline Human benchmark & Human & $97.1 \% *$ & - & - & - \\
\hline
\end{tabular}

*Raw agreement between two independent coders

\section{Results}

\section{Scene classification model results}

Table 4 presents model evaluation metrics across the model runs for both Nigeria and Guatemala. Raw agreement of the two independent coders is also provided as the human-level benchmark.

Of the four deep learning models assessed (baseline CNN, VGG16, IncetionV3, and VGG16+ Inception), the ensemble of VGG16 and InceptionV3 performed the best in Nigeria, with an accuracy of $94.4 \%$ and F1-score of $92.2 \%$. The ensemble also performed the best in Guatemala with a test set accuracy of $96.4 \%$ and F1-score of $96.5 \%$. Overall, the transfer learning models performed considerably better than the baseline $\mathrm{CNN}$, with over 93\% accuracy for both VGG16 and InceptionV3 in Nigeria (compared to $88.9 \%$ for the baseline $\mathrm{CNN}$ ) and over 95\% for both in Guatemala (compared to $93.3 \%$ for the baseline $\mathrm{CNN}$ ). Both the transfer learning models and the ensemble compared favorably to the human benchmark for Nigeria, performing better than the raw agreement (94.5 vs. 91.0\%). These models almost performed as well as the human benchmark in Guatemala (96.4 vs. 97.1\%).

As a further comparison, we created shallow classification models using GIS-derived variables from OSM and ESA to predict residential grids in Nigeria and Guatemala. Using the same grids for training and test sets as the deep learning models, our best model accuracy using the OSM + ESA variables was $80.6 \%$ in Nigeria and $93.8 \%$ in Guatemala (Table 4). In Nigeria, all models except k-nearest neighbors performed similarly, with AdaBoost and logistic regression classifiers performing slightly better than others. In Guatemala all models performed in a tight range between 92.4 and 93.8\%, although only k-nearest neighbors and AdaBoost achieved an accuracy lower than $93.8 \%$. Precision, recall, and F1-scores were also stable and consistent within country samples.

Compared to the deep learning models trained directly on images, the shallow learning models using OSM+ESA variables performed worse in both Nigeria and Guatemala. Although model accuracy was relatively 
Table 5 Test set accuracy by SGC grid size

\begin{tabular}{|c|c|c|c|c|}
\hline \multirow[t]{2}{*}{ SGC size } & \multicolumn{2}{|l|}{ Nigeria } & \multicolumn{2}{|c|}{ Guatemala } \\
\hline & Count & Accuracy & Count & Accuracy \\
\hline $50 \times 50 \mathrm{~m}$ & 74 & $98.65 \%$ & 179 & $97.21 \%$ \\
\hline $100 \times 100 \mathrm{~m}$ & 571 & $93.52 \%$ & 46 & $93.48 \%$ \\
\hline $150 \times 150 \mathrm{~m}$ & 155 & $95.48 \%$ & 0 & - \\
\hline $\begin{array}{l}\text { Test for equality } \\
\text { of proportions }\end{array}$ & \multicolumn{2}{|c|}{$\begin{array}{c}x^{2}=3.691, d f=2, \\
p \text {-value }=0.1579\end{array}$} & \multicolumn{2}{|c|}{$\begin{array}{c}x^{2}=0.595, d f=1 \\
p \text {-value }=0.4403\end{array}$} \\
\hline
\end{tabular}

close between the image and OSM + ESA models in Guatemala (93.8 vs. $96.4 \%$ ), there was a substantial difference in performance in Nigeria (80.6 vs. $94.4 \%$ ). In addition, unlike the image-based models, the OSM+ESA models greatly underperformed the human-level benchmark in Nigeria (80.6 vs. $91.0 \%$ ), while also slightly underperforming in Guatemala (93.8 vs. 97.1\%).

A possible explanation for the difference in performance of the OSM + ESA models between Nigeria and Guatemala may be because of the completeness of the OSM database for the two countries. Evidence of this comes from a recent study on road network completeness in OSM [88], which found that Nigeria had a lower estimated fraction of roads captured (36\%) than Guatemala (47\%). Although using a GIS feature model may become more reliable as developing countries get better coverage, the models trained on satellite images in this study do not suffer from this limitation.

\section{Effect of imagery source and grid area sizes}

As our data sets in Nigeria and Guatemala contain multiple image sources and grid area sizes, we test to see if accuracy on the best performing model is impacted by either sources of variation. Table 5 reports the test set accuracy across different SGC grid sizes. In Nigeria, the model was most accurate predicting $50 \mathrm{~m} \times 50 \mathrm{~m}$ grid size images (98.65\%), followed by the $150 \mathrm{~m} \times 150 \mathrm{~m}$ grid sizes $(95.48 \%)$. The model was least accurate in predicting the $100 \mathrm{~m} \times 100 \mathrm{~m}$ grid size images (93.52\%). However, as the accuracies fall in a small range, we performed a 3-sample test of proportions to account for the differences in accuracy that may occur due to chance. The test results do not provide substantial evidence to reject the null hypotheses that all the accuracy measures across SGC grid sizes are equal, given $\alpha=0.05$ $($ Chi-square $=3.691 ; p$-value $=0.1579)$. Likewise, while Guatemala also predicted $50 \mathrm{~m} \times 50 \mathrm{~m}$ grids $(97.21 \%)$ more often than $100 \mathrm{~m} \times 100 \mathrm{~m}$ grids $(93.48 \%)$, the differences in accuracy were also not statistically significant at $\alpha=0.05$ (Chi-square $=0.595 ; p$-value $=0.4403$ ).
Table 6 Test set accuracy by image source

\begin{tabular}{|c|c|c|c|c|}
\hline \multirow[t]{2}{*}{ Image source } & \multicolumn{2}{|l|}{ Nigeria } & \multicolumn{2}{|c|}{ Guatemala } \\
\hline & Count & Accuracy & Count & Accuracy \\
\hline Google & 643 & $94.25 \%$ & 179 & $97.21 \%$ \\
\hline Bing & 157 & $94.90 \%$ & 46 & $93.48 \%$ \\
\hline $\begin{array}{l}\text { Test for equality } \\
\text { of proportions }\end{array}$ & \multicolumn{2}{|c|}{$\begin{array}{c}x^{2}=0.016, d f=1 \\
p \text {-value }=0.8982\end{array}$} & \multicolumn{2}{|c|}{$\begin{array}{l}x^{2}=0.595, d f=1 \\
p \text {-value }=0.4403\end{array}$} \\
\hline
\end{tabular}

Table 6 reports the test set accuracy across different image sources. While three sets of images were provided for analysts to choose from (Google, Bing, and Esri), no images from Esri were selected for coding. In Nigeria, the model predicted near-identical accuracies across image sources $($ Google $=94.25 \%$; Bing $=94.90 \%)$. The 2-sample test of proportions also reflects this, failing to reject the null hypothesis at an $\alpha=0.05$ (Chi-square $=0.016$; $p$-value $=0.8982$ ). Surprisingly, Google was selected for all $50 \mathrm{~m} \times 50 \mathrm{~m}$ grids and Bing was chosen for all $100 \mathrm{~m} \times 100 \mathrm{~m}$ grids in Guatemala. As such, the differences in accuracy and test statistics are the same as when stratifying by grid size.

\section{Effect of training set size}

Operationalizing this method in new countries will require retraining the models with images from the new countries. To better understand the expected data annotation burden, we created learning curves to test how sensitive model performance is to training size [89]. Figure 7 shows the test set accuracy and 95\% confidence intervals for training set sizes at $10,25,50,100,250,500$, and 1000 images. Five randomly sampled training sets were created and trained for each set size, stratified to preserve the class ratios seen in the original training sets. The five trained models for each training set size were then run on the corresponding countries complete validation set to determine accuracy metrics. Although results are only presented for the pretrained VGG16 model, the learning curves showed similar trends for InceptionV3.

As expected with smaller training sizes, there is a lower average and larger variance in the accuracy for both Nigeria and Guatemala. Average accuracy increases as training size increases, from $78.2 \%(\mathrm{n}=10)$ to $91.7 \%(\mathrm{n}=1000)$ in Nigeria and $90.5 \%(\mathrm{n}=10)$ to $96.1 \%(\mathrm{n}=1000)$ in Guatemala. Although neither sets of models at these sample sizes exceed the human-level benchmarks, they do approach the baseline with modest amounts of training data. This finding both supports the robustness of transfer learning and the more practical case of portability to new areas. 


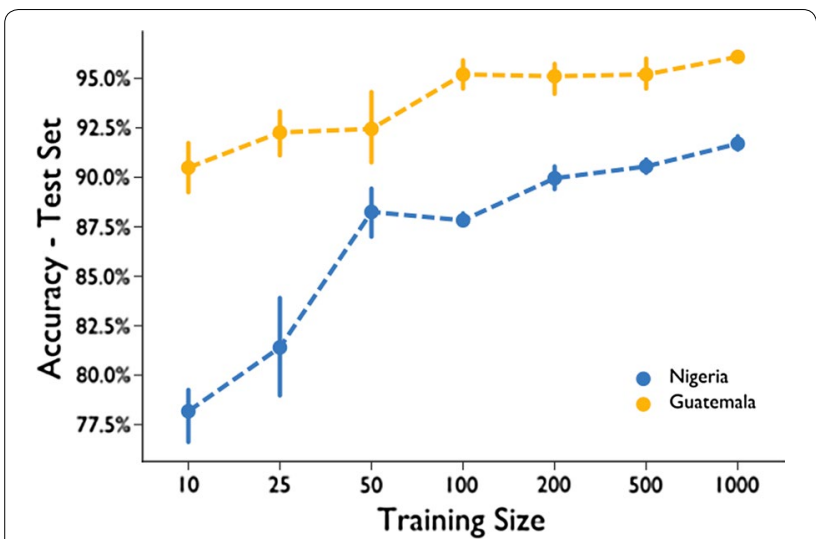

Fig. 7 Learning curves for Nigeria and Guatemala

\section{Discussion}

These findings suggest the effectiveness of deep CNNs for identifying residential grids for cluster sampling, providing an accurate and scalable way to help screen large areas with minimal data requirements. Although this method was demonstrated within the context of geosampling, the approach can be applicable to any household survey in low- and middle-income countries with a gridded population sample design. With studies showing a variety of inaccuracies for model-based population data sets at the sub-national level [26, 27], our approach could help verify, supplement, or even replace the need for gridded population estimates in certain cases.

Although little has been published on the use of scene classification for applications in survey research, our results support findings in the remote sensing literature on deep CNNs providing state-of-the-art performance on remote scene classification tasks [90], showing over 95\% overall accuracy on data sets containing anywhere from 2 [41] to 45 [91] scene categories. In particular, several studies have also documented the effectiveness of using transfer learning with CNNs pretrained on ImageNet for scene classification tasks [41-43], even though the underlying source data set does not contain satellite images. While other scene classification benchmark datasets $[91,92]$ can contain up to dozens of different categories (e.g., airplanes, stadiums, beaches, viaduct, etc.), many of these scenes are largely irrelevant for the purpose of household surveys that are only interested in residences. Of comparable studies that publish confusion matrices with scene specific accuracy metrics, residential scenes have been among the most difficult to correctly classify (Table 7). Han et al. [42] and $\mathrm{Hu}$ et al. [43], whom both also use a transfer learning approach with deep CNNs pretrained on ImageNet, found that predicted accuracy of residential classes ranged from 85 to $95 \%$, compared with our $94.5 \%$ accuracy in Nigeria and $96.4 \%$ accuracy in Guatemala. This difficulty in predicting residential scenes may be due to their high similarity to other classes or ambiguity in the definition of what is considered a "residential" scene. Especially when encountering difficult-to-define categories, collapsing classes (such as our overarching "nonresidential" class) can increase classification accuracy by simplifying the modeling task, requiring the model to distinguish only between broad, distinct categories [93]. By focusing on only two scene classes

Table 7 Residential scene classification accuracy across studies using deep CNNs transfer learning models

\begin{tabular}{|c|c|c|c|c|c|}
\hline References & Scene class & Dataset & \# Classes & Accuracy (\%) & $\begin{array}{l}\text { Relative scene } \\
\text { accuracy } \\
\text { ranking }\end{array}$ \\
\hline \multirow[t]{3}{*}{ Hu et al. [43] } & Sparse Residential & UC Merced & 21 & 85 & 19 of $21^{*}$ \\
\hline & Med. Residential & UC Merced & 21 & 85 & 19 of $21^{*}$ \\
\hline & Dense Residential & UC Merced & 21 & 90 & 17 of $21^{* *}$ \\
\hline \multirow[t]{5}{*}{ Han et al. [42] } & Sparse Residential & UC Merced & 21 & 95 & 12 of $21^{* * *}$ \\
\hline & Med. Residential & UC Merced & 21 & 90 & 19 of $21^{* * * *}$ \\
\hline & Dense Residential & UC Merced & 21 & 85 & 21 of 21 \\
\hline & Residential & SIRI-WHU & 12 & 93 & 10 of $12^{* * * * *}$ \\
\hline & Residential & WHU-RS & 19 & 88 & 19 of 19 \\
\hline \multirow[t]{2}{*}{ Chew et al. (in this study) } & Residential & Nigeria & 2 & 94.5 & NA \\
\hline & Residential & Guatemala & 2 & 96.4 & NA \\
\hline
\end{tabular}

*Medium and Sparse residential tied for 19th/20th place

**Tied with "intersection" for $17 \mathrm{th} / 18$ th place

***Tied with seven other classes for 12th-18th place

****Tied with "storage tank" for 19th/20th place

*****Tied with "idle land" for 10 th/11 th place 
in our modeling, survey researchers not only benefit from a potentially higher accuracy model than if they included additional scenes, but the scenes included are only those relevant for downstream analysis.

In addition to providing survey research teams with a method for screening residential areas, our work also provides contributions to the larger scene classification literature. While deep CNNs have been effective on scene classification tasks ranging in spatial resolutions (2-m resolution in SIRI-WHU dataset [94] to 1 -ft resolution in UC Merced dataset) [95], few studies have reported applying deep CNN scene classification models to datasets containing multiple spatial resolutions as found in our data set. We do not find statistically significant differences in accuracies between grid area sizes and image sources, suggesting that deep CNN models can perform well on image datasets that contain heterogeneous properties and that may resemble data collected by survey research and implementation teams on projects in developing countries. Additionally, most other benchmark scene classification datasets contain images from developed areas, such as the United States [40, 95], Europe [91], and urban areas in China [96], rather than low- and middle-income countries. By extending scene classification to Nigeria and Guatemala, we provide additional evidence that methods shown to be effective in developed nations also apply to developing nations where data quality and availability is generally worse.

While initial results are promising, future work could expand the training set to include a larger and more diverse geographic scope to better understand how the method generalizes across developing nations. Furthermore, since SGC images are localized within PGCs, our training samples are highly clustered geographically. This is appropriate for our use case; however, future research could validate if the high accuracy found in this study applies when predicting random SGC grids within a country. Extended analyses could also examine the extent of spatial autocorrelation among residential grids and assess if methods that explicitly model this dependence (e.g., Markov random fields) can help improve model accuracy. In future work, deep learning models could also be applied at the PGC level. Although this could reduce the existing multistep process that is required to implement manual residential screening down to a single step, it is unclear whether the heterogeneity within the larger PGCs would impact the effectiveness of the method.

One limitation of our study was that our nonresidential grids contained a variety of landscapes, including agricultural, forested, and predominately commercial areas without residencies. While we argue that focusing the problem specifically on residential versus nonresidential will likely be preferred for gridded population sampling for household surveys, future research can be directed toward better understanding whether creating more granular scene categories for nonresidential grids can refine the screening process, particularly in helping disambiguate areas in the built environment (residential vs. commercial). This option would need to be balanced against the additional labelling burden of coders needing to choose among multiple classes. The current geosampling methodology only requires knowing whether residential buildings are present in the area. However, the task could be reframed as an object detection problem with the objective of identifying the number of buildings in a grid instead of just the presence or absence of residential buildings. The extension of this work to an object detection task could facilitate the estimation of population estimates for SGCs or may allow direct selection of households from aerial images.

Lastly, although we present these metrics as an assessment of how well our models compare to human performance on this task, we recognize that the specific values for the human-level benchmark are only representative of the coders recruited to assist for this study. Coders with different levels of experience, skill, and conscientiousness than ours would likely produce different results. Additionally, these numbers represent the disagreement across both training and test sets in Nigeria and Guatemala, whereas the model predictions are only assessed on the test sets. Nonetheless, these ballpark figures do provide us greater assurance of this method's merits compared to the status quo and much needed context to the raw model performance metrics.

\section{Conclusion}

Using deep CNNs, we demonstrated that we can correctly classify whether areas are residential or nonresidential from aerial satellite images, meeting or exceeding a human-level benchmark in both Nigeria and Guatemala. Not only does this capability reduce the manual resources and calendar time needed for labelling images on future geosampling projects, but it will also improve calculation of probabilities of selection at GIS sampling stages by avoiding unnecessary assumptions about the population distribution. Our findings also suggest that this approach can work well in new areas with relatively modest amounts of training data. Lastly, in areas where GIS variables from data sources like OSM are well populated, using GIS derived feature variables can also accurately detect whether a grid is residential or nonresidential. However, our findings suggest that using CNNs trained on satellite images work even when crowdsourced spatial data sets are not well populated or maintained. 


\begin{abstract}
Abbreviations
CNNs: convolutional neural networks; ESA: European Space Agency; GEOBIA Geographic Object-based Image Analysis; GIS: geographic information systems; GUI: graphical user interface; OSM: OpenStreetMaps; RMSE: root mean squared error; PGC: primary grid cells; ReLU: rectified linear unit; SGC: secondary grid cells.
\end{abstract}

\section{Authors' contributions}

$\mathrm{RC}$ led the conception and design of the research agenda for the machine learning methods and validation, contributed substantially to the analysis and interpretation of data, and led the authoring, revision, and submission of the manuscript. SA made substantial contributions to conception and design of the geosampling methodology and research analysis plan, contributed to the manuscript draft, and provided critical guidance on survey statistics and sampling. KJ made substantial contributions to analysis and interpretation of the machine learning methodology and provided critical manuscript revisions. JU made substantial contributions to revising the manuscript critically for important intellectual content and provided critical guidance on survey statistics and sampling. JC made substantial contributions to acquiring and processing the satellite imagery data and provided critical manuscript revisions. JA made contributions to the acquisition of data and contributed to the manuscript draft and revisions. MB made contributions to the acquisition of the OSM + ESA data (including feature creation) and contributed to the conception and study design. All authors read and approved the final manuscript.

\section{Author details}

${ }^{1}$ Center for Data Science, RTI International, 3040 East Cornwallis Road, Research Triangle Park, NC, USA. ${ }^{2}$ Division for Statistical and Data Sciences, RTI International, 3040 East Cornwallis Road, Research Triangle Park, NC, USA. ${ }^{3}$ Geospatial Science and Technology Program, RTI International, 3040 East Cornwallis Road, Research Triangle Park, NC, USA.

\section{Acknowledgements}

We would like to thank Sam Goree for his initial machine learning modeling on the OSM + ESA features and Vivian Chen for her early exploratory work during her internship and for the valued assistance with manual annotation, Additionally, we thank Gayle Bieler and Karol Krotki for reading drafts and providing thoughtful edits.

\section{Competing interests}

The authors declare that they have no competing interests.

\section{Availability of data and materials}

The data sets used and/or analyzed during the current study are available from the corresponding author on reasonable request.

\section{Consent for publication}

Not applicable.

\section{Ethics approval and consent to participate}

Not applicable.

\section{Funding}

This research was funded through RTI International Strategic Investment Funds.

\section{Publisher's Note}

Springer Nature remains neutral with regard to jurisdictional claims in published maps and institutional affiliations.

Received: 26 January 2018 Accepted: 3 May 2018

Published online: 09 May 2018

\section{References}

1. Harkness J. Guidelines for best practice in cross-cultural surveys. Michigan: University of Michigan; 2011.
2. Kamanga A, Renn S, Pollard D, Bridges DJ, Chirwa B, Pinchoff J, et al. Open-source satellite enumeration to map households: planning and targeting indoor residual spraying for malaria. Malar J. 2015;14:345.

3. Bauer J. Selection errors of random route samples. Sociol Methods Res. 2014:43:519-44.

4. Bauer J. Biases in random route surveys. J Surv Stat Methodol. 2016:4:263-87.

5. Boyd HW, Westfall R. Interviewers as a source of error in surveys. J Mark. 1955:19:311-24.

6. Boyd HW, Westfall R. Interviewer bias once more revisited. J Mark Res. 1970;7:249-53.

7. Boyd HW Jr, Westfall R. Interviewer bias revisited. J Mark Res. 1965:2:58-63.

8. Eckman S, Kreuter F. Confirmation bias in housing unit listing. Public Opin Q. 2011;75:139-50.

9. Hanson $\mathrm{RH}$, Marks ES. Influence of the interviewer on the accuracy of survey results. J Am Stat Assoc. 1958;53:635-55.

10. Hoag WJ, Allerbeck KR. Interviewer and situation effects in surveys: a loglinear analysis. J Sociol. 1981;10:413-26.

11. Manheimer $\mathrm{D}, \mathrm{Hyman} \mathrm{H}$. Interviewer performance in area sampling. Public Opin Q. 1949;13:83-92.

12. O'Muircheartaigh C, Campanelli P. The relative impact of interviewer effects and sample design effects on survey precision. J R Stat Soc Ser A (Stat Soc). 1998;161:63-77.

13. Schnell R, Kreuter F. Separating interviewer and sampling-point effects. J Off Stat. 2005;21:389.

14. Amer S. Geo-sampling: from design to implementation. Presented at AAPOR: Hollywood, FL; 2015. http://www.aapor.org/AAPOR_Main/media /AM15/Abstract_Book_Final.pdf.

15. Elsey $H$, Thomson DR, Lin RY, Maharjan U, Agarwal S, Newell J. Addressing inequities in urban health: Do decision-makers have the data they need? Report from the Urban Health Data Special Session at International Conference on Urban Health Dhaka 2015. J Urban Health. 2016;93:526-37.

16. Galway LP, Bell N, Al Shatari SA, Hagopian A, Burnham G, Flaxman A, et al. A two-stage cluster sampling method using gridded population data, a GIS, and Google EarthTM imagery in a population-based mortality survey in Iraq. Int J Health Geogr. 2012;11:12.

17. Hagopian A, Flaxman AD, Takaro TK, Esa Al Shatari SA, Rajaratnam J, Becker S, et al. Mortality in Iraq associated with the 2003-2011 war and occupation: findings from a national cluster sample survey by the university collaborative Iraq Mortality Study. PLoS Med. 2013;10:e1001533.

18. Muñoz J, Langeraar E. A census independent sampling strategy for a household survey in Myanmar. 2013. Available at: bit. ly/TU94rr.

19. Shields T, Pinchoff J, Lubinda J, Hamapumbu H, Searle K, Kobayashi T, et al. Spatial and temporal changes in household structure locations using high-resolution satellite imagery for population assessment: an analysis of household locations in southern Zambia between 2006 and 2011. Geospatial Health. 2016;11:410.

20. Sollom R, Richards AK, Parmar P, Mullany LC, Lian SB, lacopino V, et al. Health and human rights in Chin State, Western Burma: a populationbased assessment using multistaged household cluster sampling. PLoS Med. 2011;8:e1001007.

21. Tatem AJ, Thomson DR, Stevens FR, Castro MC, Ruktanonchai NW. GridSample: an R package to generate household survey primary sampling units (PSUs) from gridded population data. Int J Health Geogr. 2017:16:25

22. Thomson DR, Hadley MB, Greenough PG, Castro MC. Modelling strategic interventions in a population with a total fertility rate of 8.3: a crosssectional study of Idjwi Island, DRC. BMC Public Health. 2012;12:959.

23. Oak Ridge National Laboratory. LandScan Global Population Dataset 2013. Oak Ridge: Oak Ridge National Laboratory; 2014.

24. Kish L. Survey sampling. New York: Wiley; 1965.

25. Rose A, McKee J, Weber E, Bhaduri BL. Geoscience meets social science: A flexible data driven approach for developing high resolution population datasets at global scale. In: AGU Fall Meeting Abstracts. 2017.

26. Linard C, Alegana VA, Noor AM, Snow RW, Tatem AJ. A high resolution spatial population database of Somalia for disease risk mapping. Int J Health Geogr. 2010;9:45.

27. Tatem AJ, Noor AM, Hay SI. Assessing the accuracy of satellite derived global and national urban maps in Kenya. Remote Sens Environ. 2005:96:87-97. 
28. Himelein K, Eckman S, Murray S. Sampling nomads: a new technique for remote, hard-to-reach, and mobile populations. J Off Stat. 2014;30:191-213.

29. Himelein K, Eckman S, Murray S, Bauer J. Second-stage sampling for conflict areas. World Bank Group. Policy Research Working Paper 7617; 2016.

30. Byrne GF, Crapper PF, Mayo KK. Monitoring land-cover change by principal component analysis of multitemporal Landsat data. Remote Sens Environ. 1980;10:175-84.

31. Congalton RG, Oderwald RG, Mead RA. Assessing Landsat classification accuracy using discrete multivariate analysis statistical techniques. Photogramm Eng Remote Sens. 1983;49:1671-8.

32. Tucker CJ, Townshend JR, Goff TE. African land-cover classification using satellite data. Sci Transl Med. 1985;227:369-75.

33. Blaschke T. Object based image analysis for remote sensing. ISPRS J Photogram Remote Sens. 2010;65:2-16.

34. Blaschke T, Hay GJ, Kelly M, Lang S, Hofmann P, Addink E, et al. Geographic object-based image analysis-towards a new paradigm. ISPRS J Photogramm Remote Sens. 2014;87:180-91.

35. Hay GJ, Castilla G. Geographic Object-Based Image Analysis (GEOBIA): A new name for a new discipline. Object-Based Image Analysis. 2008. p. 75-89.

36. LeCun Y, Bengio Y, Hinton G. Deep learning. Nat. Biotech. 2015:521:436-44

37. Mnih V, Hinton GE. Learning to label aerial images from noisy data. In: Proceedings of the 29th international conference on machine learning (ICML-12). 2012. p. 567-74.

38. Längkvist M, Kiselev A, Alirezaie M, Loutfi A. Classification and segmentation of satellite orthoimagery using convolutional neural networks. Remote Sens. 2016;8:329.

39. Jean N, Burke M, Xie M, Davis WM, Lobell DB, Ermon S. Combining satellite imagery and machine learning to predict poverty. Sci Transl Med. 2016:353:790-4

40. Basu S, Ganguly S, Mukhopadhyay S, DiBiano R, Karki M, Nemani R. Deepsat: A learning framework for satellite imagery. In: Proceedings of the 23rd SIGSPATIAL international conference on advances in geographic information systems ACM. 2015, November, p. 37.

41. Castelluccio M, Poggi G, Sansone C, Verdoliva L. Land use classification in remote sensing images by convolutional neural networks. arXiv preprint arXiv. 2015.

42. Han X, Zhong Y, Cao L, Zhang L. Pre-trained AlexNet architecture with pyramid pooling and supervision for high spatial resolution remote sensing image scene classification. Remote Sens. 2017;9:848.

43. Hu F, Xia GS, Hu J, Zhang L. Transferring deep convolutional neural networks for the scene classification of high-resolution remote sensing imagery. Remote Sens. 2015;7:14680-707.

44. Zhong Y, Fei F, Liu Y, Zhao B, Jiao H, Zhang L. SatCNN: satellite image dataset classification using agile convolutional neural networks. Remote Sens Lett. 2017;8:136-45.

45. Carletta J. Assessing agreement on classification tasks: the kappa statistic. Comput Linguist. 1996;22:249-54.

46. Cohen J. A coefficient of agreement for nominal scales. Educ Psychol Meas. 1960;20:37-46.

47. Landis JR, Koch GG. The measurement of observer agreement for categorical data. Biometrics. 1977;33:159.

48. Spitznagel EL, Helzer JE. A proposed solution to the base rate problem in the kappa statistic. Arch Gen Psychiatry. 1985:42:725-8.

49. Uebersax JS. Diversity of decision-making models and the measurement of interrater agreement. Psychol Bull. 1987;101:140.

50. Pontius RG Jr, Millones M. Death to Kappa: birth of quantity disagreement and allocation disagreement for accuracy assessment. Int J Remote Sens. 2011:32:4407-29.

51. Olofsson P, Foody GM, Herold M, Stehman SV, Woodcock CE, Wulder MA. Good practices for estimating area and assessing accuracy of land change. Remote Sens Environ. 2014;148:42-57.

52. Szegedy C, Vanhoucke V, loffe S, Shlens J, Wojna Z. Rethinking the inception architecture for computer vision. In: Proceedings of the IEEE conference on computer vision and pattern recognition. 2016. p. 2818-26.

53. Simonyan K, Zisserman A. Very deep convolutional networks for largescale image recognition. arXiv preprint arXiv. 2014.

54. He K, Zhang X, Ren S, Sun J. Deep residual learning for image recognition. arXiv preprint. 2015. http://arxiv.org/abs/1512.03385.
55. Russakovsky O, Deng J, Su H, Krause J, Satheesh S, Ma S, et al. ImageNet large scale visual recognition challenge (Tech. Rep.). 2015.

56. Szegedy C, Liu W, Jia Y, Sermanet P, Reed S, Anguelov D, et al. Going deeper with convolutions. arXiv preprint. 2014. http://arxiv.org/ abs/1409.4842.

57. Graves A, Mohamed A-R, Hinton G. Speech recognition with deep recurrent neural networks. In Acoustics, speech and signal processing (ICASSP), 2013 IEEE international conference on acoustics, speech and signal processing - proceedings. 2013. p. 6645-9.

58. Weng C, Yu D, Watanabe S, Juang B-HF. Recurrent deep neural networks for robust speech recognition. In ICASSP, IEEE international conference on acoustics, speech and signal processing_-proceedings. 2014. p. 5532-6.

59. Guo X, Singh $\mathrm{S}$, Lee $H$, Lewis RL, Wang X. Deep learning for real-time Atari game play using offline Monte-Carlo tree search planning. In: Advances in neural information processing systems. 2014. p. 3338-46.

60. Schaul T, Quan J, Antonoglou I, Silver D. Prioritized experience replay. In International conference on learning representations (ICLR). 2016.

61. Stadie BC, Levine S, Abbeel P. Incentivizing exploration in reinforcement learning with deep predictive models. arXiv preprint. 2016. http://arxiv .org/abs/1507.00814

62. European Space Agency. GlobCover Land Cover v2 2008 database. European Space Agency GlobCover Project, led by MEDIAS-France. 2008. http://ionia1.esrin.esa.int/index.asp.

63. Wolpert $\mathrm{DH}$. The lack of a priori distinctions between learning algorithms. Neural Comput. 1996;8:1341-90.

64. Reed TR, Dubuf JH. A review of recent texture segmentation and feature extraction techniques. CVGIP: Image Understand. 1993;57:359-72.

65. Yang M, Kpalma K, Ronsin J. A survey of shape feature extraction techniques. In: Pattern recognition, IN-TECH. 2008. p. 43-90.

66. Boureau YL, Ponce J, LeCun Y. A theoretical analysis of feature pooling in visual recognition. In Proceedings of the 27th international conference on machine learning (ICML-10). 2010. p. 111-1.

67. Krizhevsky A, Sutskever I, Hinton GE. Imagenet classification with deep convolutional neural networks. In: Advances in neural information processing systems. 2012. p. 1097-105.

68. Bishop CM. Pattern recognition and machine learning (information science and statistics). New York: Springer; 2006.

69. Srivastava N, Hinton GE, Krizhevsky A, Sutskever I, Salakhutdinov R. Dropout: a simple way to prevent neural networks from overfitting. J Mach Learn Res. 2014;15:1929-58.

70. Pan SJ, Yang Q. A survey on transfer learning. IEEE Trans Knowl Data Eng. 2010:22:1345-59.

71. Sharif Razavian A, Azizpour H, Sullivan J, Carlsson S. CNN features offthe-shelf: an astounding baseline for recognition. In: Proceedings of the IEEE conference on computer vision and pattern recognition workshops. 2014. p. 806-13.

72. Yosinski J, Clune J, Bengio Y, Lipson H. How transferable are features in deep neural networks? In: Advances in neural information processing systems. 2014. p. 3320-8

73. Deng J, Dong W, Socher R, Li LJ, Li K, Fei-Fei L. Imagenet: A large-scale hierarchical image database. In Computer vision and pattern recognition, 2009 (CVPR 2009) IEEE conference; June. 2009. p. 248-55.

74. Miller GA. WordNet: a lexical database for English. Commun ACM. 1995:38:39-41.

75. Shin HC, Roth HR, Gao M, Lu L, Xu Z, Nogues I, et al. Deep convolutional neural networks for computer-aided detection: cNN architectures, dataset characteristics and transfer learning. IEEE Trans Med Imaging. 2016:35:1285-98

76. Marmanis D, Datcu M, Esch T, Stilla U. Deep learning earth observation classification using ImageNet pretrained networks. IEEE Geosci Remote Sens Lett. 2016;13:105-9.

77. Chollet F. Deep learning with python. 1st ed. Greenwich: Manning Publications Co.; 2017

78. Bayramoglu N, Heikkilä J. Transfer learning for cell nuclei classification in histopathology images. In: European conference on computer vision. Cham: Springer; 2016. p. 532-9.

79. Park J, Javier RJ, Moon T, Kim Y. Micro-Doppler based classification of human aquatic activities via transfer learning of convolutional neural networks. Sensors. 2016:16:1990. 
80. Bargoti S, Underwood J. Deep fruit detection in orchards. In 2017 IEEE international conference robotics and automation (ICRA); May. 2017. p. 3626-33.

81. Chollet F, others. Keras. Github repository. 2015. https://github.com/fchol let/keras.

82. Yu D, Seltzer ML. Improved bottleneck features using pretrained deep neural networks. In: Twelfth annual conference of the international speech communication association. 2011.

83. Opitz DW, Maclin R. Popular ensemble methods: an empirical study. J Artif Intell Res (JAIR). 1999;11:169-98.

84. Polikar R. Ensemble based systems in decision making. IEEE Circuits Syst Mag. 2006;6:21-45.

85. Rokach L. Ensemble-based classifiers. Artif Intell Rev. 2010;33:1-39.

86. OpenStreetMap contributors. Planet dump retrieved from https://plane t.openstreetmap.org; 2017.

87. Pedregosa F, Varoquaux G, Gramfort A, Michel V, Thirion B, Grisel O, et al. Scikit-learn: machine learning in Python. J Mach Learn Res. 2011;12:2825-30.

88. Barrington-Leigh C, Millard-Ball A. The world's user-generated road map is more than 80\% complete. PLoS ONE. 2017;12:e0180698.

89. Amari SI, Fujita N, Shinomoto S. Four types of learning curves. Neural Comput. 1992:4:605-18.
90. Zhu XX, Tuia D, Mou L, Xia GS, Zhang L, Xu F, et al. Deep learning in remote sensing: a comprehensive review and list of resources. IEEE Geosci Remote Sens Mag. 2017;5:8-36.

91. Cheng G, Han J, Lu X. Remote sensing image scene classification: benchmark and state of the art. Proc IEEE. 2017:105:1865-83.

92. Xia GS, Hu J, Hu F, Shi B, Bai X, Zhong Y, et al. AID: a benchmark data set for performance evaluation of aerial scene classification. IEEE Trans Geosc Remote Sens. 2017;55:3965-81.

93. Strahler AH, Boschetti L, Foody GM, Friedl MA, Hansen MC, Herold M, et al. Global land cover validation: recommendations for evaluation and accuracy assessment of global land cover maps. Luxembourg: European Communities; 2006. p. 51.

94. Liu Y, Zhong Y, Fei F, Zhang L. Scene semantic classification based on random-scale stretched convolutional neural network for high-spatial resolution remote sensing imagery. In: 2016 IEEE international geoscience and remote sensing symposium (IGARSS); 2016. p. 763-6.

95. Yang Y, Newsam S. Bag-of-visual-words and spatial extensions for land-use classification. In: ACM SIGSPATIAL international conference on advances in geographic information systems (ACM GIS). 2010.

96. Zhu Q, Zhong Y, Zhao B, Xia G-S, Zhang L. Bag-of-visual-words scene classifier with local and global features for high spatial resolution remote sensing imagery. IEEE Geosci Remote Sens Lett. 2016;13:747-51.
Ready to submit your research? Choose BMC and benefit from:

- fast, convenient online submission

- thorough peer review by experienced researchers in your field

- rapid publication on acceptance

- support for research data, including large and complex data types

- gold Open Access which fosters wider collaboration and increased citations

- maximum visibility for your research: over 100M website views per year

At BMC, research is always in progress.

Learn more biomedcentral.com/submissions 